\section{Associação entre mortalidade e estado marital: uma análise para idosos residentes no Município de São Paulo, Brasil, Estudo SABE, 2000 e 2006}

\author{
Association between marital status and mortality \\ among elderly residents in São Paulo City, Brazil, \\ SABE Study, 2000 and 2006
}

\begin{abstract}
Asociación entre el estado civil y la mortalidad: un análisis de los ancianos residentes en São Paulo, Brasil, el Estudio SABE, 2000 y 2006
\end{abstract}

\author{
1 Universidade Católica de \\ Brasília, Brasília, Brasil. \\ 2 Centro de Desenvolvimento \\ e Planejamento Regional, \\ Universidade Federal de \\ Minas Gerais, Belo Horizonte, \\ Brasil. \\ 3 Escola de Enfermagem, \\ Universidade de São Paulo, \\ São Paulo, Brasil. \\ ${ }^{4}$ Faculdade de Saúde \\ Pública, Universidade de \\ São Paulo, São Paulo, Brasil. \\ Correspondência \\ M. M. F. Gomes \\ Universidade Católica de \\ Brasília. \\ QE 28, conjunto R, casa 15, \\ Guará II, Brasília, DF \\ 71060-182, Brasil. \\ mariliamfg@gmail.com
}

\begin{abstract}
This study aimed to analyze the association between mortality and marital status in the elderly population (60 years and older) in São Paulo, Brazil. The authors used data from the SABE Study (Health, Well-Being, and Aging) from 2000 and 2006. Statistical analysis used Poisson regression, considering variation in time of mortality risk. The mortality rate among single elderly males was $61 \%$ higher than among married men. Separation or divorce and widowhood increased the odds of death in elderly women (separated/ divorced women and widows showed mortality rates $82 \%$ and $35 \%$ higher, respectively, than married women). It is hoped that the current study will improve our understanding of factors associated with survival in the elderly, in addition to supporting health policies for this population group.
\end{abstract}

Mortality; Aged; Marital Status

\author{
Marília Miranda Forte Gomes 1 \\ Cássio M. Turra ${ }^{2}$ \\ Moema Gonçalves Bueno Fígoli 2 \\ Yeda A. O. Duarte 3 \\ Maria Lúcia Lebrão 4
}

\section{Resumo}

Este trabalho tem como objetivo analisar, para a população com 60 anos e mais, residente no Município de São Paulo, Brasil, a associação entre mortalidade e estado marital. Para atingir o objetivo proposto, foram utilizados os dados do Estudo SABE: Saúde, Bem-estar e Envelhecimento (SABE), realizado nos anos 2000 e 2006, e modelos de Regressão de Poisson foram estimados, levando-se, em consideração, a variação do tempo de risco de morte. No geral, os resultados indicam que, entre os idosos paulistanos do sexo masculino, a taxa de mortalidade dos solteiros é 61\% maior que a taxa de mortalidade observada para os casados. Por sua vez, a separação/divórcio ou a viuvez parece elevar a chance de morte das mulheres idosas analisadas. No geral, idosas separadas e viúvas apresentaram taxas de mortalidade $82 \%$ e 35\% maiores que a observada para as casadas. Espera-se que este trabalho possa contribuir para um melhor entendimento dos fatores associados à sobrevivência dos idosos, além de subsidiar políticas de saúde voltadas para esse contingente populacional.

Mortalidade; Idoso; Estado Civil 


\section{Introdução}

Os expressivos ganhos de longevidade das últimas décadas trouxeram à tona um desconhecimento preocupante sobre o comportamento corrente e futuro da mortalidade de adultos e de idosos no país (Turra CM. O Padrão de Mortalidade Adulta no Brasil: Implicações dos Erros de Declaração de Idade. Projeto de pesquisa. CNPq; 2007). Segundo Vaupel 1 e Camarano et al. 2, há indícios de que a mortalidade em idades avançadas continue diminuindo em todo o mundo, inclusive no Brasil. Uma vez que os ganhos não são igualmente distribuídos entre os diversos subgrupos da população, é importante examinar quais são os fatores associados à longevidade. Nesse contexto, alguns estudos 3,4,5,6 destacam que o estado marital desempenha um papel significativo na determinação dos diferenciais de mortalidade adulta. No geral, em países desenvolvidos, os indivíduos casados apresentam maior sobrevivência quando comparados aos divorciados/separados, viúvos e nunca casados, e essas diferenças são mais elevadas entre os homens e diminuem com o avançar da idade 3,4,7,8,9.

Na tentativa de explicar o diferencial de mortalidade por estado marital, a literatura destaca a existência de duas hipóteses. A primeira é a proteção do casamento: um conjunto de mecanismos causais, constituído por fatores ambientais, econômicos, sociais e psicológicos, contribui para que o indivíduo casado tenha maiores chances de sobrevivência do que indivíduos não casados. A segunda hipótese é baseada na premissa de que o casamento é seletivo, elegendo assim os indivíduos mais saudáveis tanto física quanto psicologicamente $3,7,10$.

Independente de qual hipótese é a que melhor explica a relação entre estado marital e mortalidade, há várias razões que justificam a estimação desses diferenciais no Brasil. Em primeiro lugar, estudos sobre a mortalidade de subgrupos da população permitem a formulação de hipóteses mais robustas para a projeção da mortalidade adulta de um país ou região ${ }^{3,11}$. Além disso, em um cenário de mudanças significativas no padrão dos arranjos domiciliares, como o que tem sido observado no caso brasileiro, caracterizado pelo aumento na prevalência de domicílios unipessoais nas idades mais velhas 12 , o estudo de diferenciais de mortalidade por estado marital torna-se especialmente relevante. Nesse contexto, quanto maior for o conhecimento sobre os riscos de morte de idosos casados e não casados, melhores são as chances de identificação e assistência dos indivíduos mais vulneráveis na população 13. Por exemplo, se há evidências de uma maior mortalidade entre idosos enlutados, polí- ticas que têm como objetivo o acompanhamento e/ou intervenções logo após o falecimento do cônjuge poderiam contribuir para a qualidade da saúde física e mental desses idosos 11.

Apesar da importância do tema, pouco se sabe sobre os diferenciais de mortalidade por estado marital em países em desenvolvimento, especialmente para grupos etários mais avançados 6 . Uma parte desse desconhecimento é explicada tanto pela ausência quanto pela inconsistência das informações coletadas nas fontes de dados tradicionais, isto é, nos registros vitais e censos demográficos. Este artigo se beneficia de um novo estudo de painel com informações sobre a sobrevivência de seus participantes para examinar os diferenciais de mortalidade por estado marital entre idosos residentes no Município de São Paulo. Além de estimar a relação direta entre estado marital e mortalidade, o trabalho analisa o papel de variáveis mediadoras nessa associação, incluindo variáveis demográficas, socioeconômicas e de saúde.

\section{Métodos}

O presente artigo utiliza dados do Estudo $S A B E$ : Saúde, Bem-estar e Envelhecimento realizado nos anos 2000 e 2006, no Município de São Paulo, com base em uma amostra representativa da população urbana de 60 anos e mais. A amostra do SABE foi constituída em duas fases distintas. Inicialmente, definiu-se que seriam visitados, no mínimo, 5.882 domicílios particulares permanentes. O sorteio desses domicílios foi realizado com base no método de amostragem por conglomerados, em dois estágios. No primeiro estágio, utilizou-se o cadastro permanente de 72 setores censitários, disponível no Departamento de Epidemiologia da Faculdade de Saúde Pública da Universidade de São Paulo, constituído a partir do cadastro da Pesquisa Nacional por Amostra de Domicílios (PNAD). No segundo estágio, calculou-se o número mínimo de domicílios que deveriam ser visitados, por setor, dividindo-se o número total de domicílios pelo número de setores censitários determinados no primeiro estágio $(5.882 / 72=81,69$ que foi aproximado para 90). Posteriormente, dividiu-se o total de endereços de cada setor censitário em segmentos de 10 domicílios, e, em cada setor, foram sorteados nove segmentos. Assim, 90 domicílios foram visitados em cada setor censitário, e todos os indivíduos considerados elegíveis, segundo os objetivos da pesquisa, foram identificados e convidados a participar das entrevistas. Ao final dessa primeira fase, 1.568 idosos foram entrevistados 14,15. 
Na segunda fase do processo amostral, foram acrescidos 575 idosos com 75 anos ou mais, visando compensar o efeito da mortalidade nesse subgrupo populacional e completar o número desejado de entrevistas nessa faixa etária. Essa segunda parte da amostra foi realizada por meio da localização dos respectivos idosos residentes em moradias próximas aos setores selecionados ou, no máximo, dentro dos limites dos distritos aos quais pertenciam os setores sorteados 14,15. A soma dos entrevistados na primeira e na segunda fase do processo de amostragem resultou na amostra final representada por 2.143 idosos não institucionalizados e residentes nas áreas urbanas do Município de São Paulo.

Os pesos amostrais finais foram ajustados levando-se, em consideração, as duas fases do processo de amostragem, a composição da população segundo faixa etária e sexo e o tamanho da população com 60 anos e mais recenseada pelo Instituto Brasileiro de Geografia e Estatística (IBGE) na contagem populacional de 1996. Uma descrição mais detalhada de como esses pesos foram calculados é apresentada no trabalho de Silva 14. Seguindo a recomendação de Silva 14 , não só os pesos amostrais, mas, também, o plano amostral delineado para o Estudo SABE foram considerados no cálculo das estimativas dos modelos apresentados neste artigo. Para tanto, utilizaram-se rotinas específicas para o tratamento de dados provenientes de amostras complexas disponíveis no programa Stata, versão 9.0 (Stata Corp., College Station, Estados Unidos).

Entre os 2.143 idosos que participaram da primeira rodada do estudo em 2000, 1.115 foram reentrevistados na segunda rodada da pesquisa, entre julho de 2006 e dezembro de 2007. Os in- divíduos não entrevistados na segunda rodada (1.028) foram classificados nas seguintes categorias: óbitos, institucionalizações, mudança de residência, recusas e idosos que não foram encontrados. Com base nessa classificação, foi possível determinar a condição de sobrevivência de cada idoso ao final do período de observação (Tabela 1). A amostra analisada neste artigo incluiu 1.355 idosos sobreviventes e 649 óbitos, totalizando 2.004 idosos. A diferença de 139 idosos em relação à amostra inicial é composta por indivíduos que foram entrevistados em 2000, mas para os quais não foi possível determinar sua condição de sobrevivência em 2006. A exclusão desses indivíduos "não localizados" não altera o padrão de distribuição das variáveis utilizadas nos nossos modelos de análise, segundo resultados de testes qui-quadrado (valor de $\mathrm{p}<0,01$ ).

Para analisar a relação entre mortalidade e estado marital, foram selecionadas 15 variáveis explicativas, a partir do questionário da SABE, que correspondem a dimensões comumente examinadas na literatura para a avaliação das hipóteses de proteção e seletividade do casamento. São elas:

- Características demográficas básicas: estado marital [categorizada como casado (referência), divorciado/separado, viúvo e solteiro]; idade [dividida em três grupos: 60 a 69 anos ou sexagenários (referência), 70 a 79 anos ou septuagenários e 80 anos e mais];

- Condições de saúde: saúde autorreportada [excelente/muito boa/boa (referência) e regular/ ruim]; número de doenças crônicas [nenhuma (referência), uma ou duas, três ou mais]; presença de incapacidade para realizar atividades básicas da vida diária - ABVD [nenhuma (referência), pelo menos uma incapacidade];

Tabela 1

Classificação da amostra, segundo status de sobrevivência, a partir da condição na segunda rodada da pesquisa. Município de São Paulo, Brasil, 2006.

\begin{tabular}{lccc}
\hline Estado final em 2006 & Idosos & $\%$ & $\begin{array}{c}\text { Estado final considerado } \\
\text { na análise }\end{array}$ \\
\hline Reentrevistado & 1.115 & 52,0 & Sobrevivente \\
Óbito & 649 & 30,3 & Óbito \\
Não localizado & 139 & 6,5 & Missing \\
Mudança - outro município & 51 & 2,4 & Sobrevivente \\
Institucionalizado & 11 & 0,5 & Sobrevivente \\
Recusa & 178 & 8,3 & Sobrevivente \\
Total & 2.143 & 100 & \\
\hline
\end{tabular}

Fonte: Estudo SABE, 2000 e 2006. 
- Status socioeconômico: nível de escolaridade, segundo anos de estudo [nenhum (referência), 1 a 7 anos, 8 anos ou mais]; se tem seguro/plano privado de saúde [sim (referência)]; se tem moradia própria [sim (referência), não]; se fez uso de serviços de saúde nos últimos 12 meses [usou (referência), não usou]; estado nutricional [bem nutrido (referência), ou não está bem nutrido];

- Meio social: se o idoso teve alguma participação comunitária no último ano (não participou [referência], participou);

- Estilo de vida: hábito de fumar [fuma atualmente (referência), já fumou, mas não fuma mais, nunca fumou]; se faz ou não uso de bebida alcoólica [sim (referência), não]; prática de atividades físicas [sim (referência), não].

Cabe ressaltar que todas as variáveis explicativas consideradas referem-se a informações declaradas pelo idoso em 2000, na primeira entrevista (linha de base). Essa estratégia de investigação foi adotada porque não se dispõe dessas informações atualizadas para os idosos que faleceram ou para aqueles que não foram reentrevistados em 2006 (889 idosos, conforme Tabela 1).

Para analisar a associação do estado marital com a mortalidade, controlando pelas variáveis descritas anteriormente, foram ajustados modelos de regressão de Poisson. O modelo de Poisson estima o número esperado de mortes durante o período de observação como uma função do tempo de exposição ao risco de morte e das variáveis explicativas selecionadas (Equação 1) 16. Uma vantagem de sua aplicação, em relação a outros modelos de análise de sobrevivência, é que é possível calcular as taxas de mortalidade para cada subgrupo, diretamente, a partir do exponencial dos coeficientes estimados na regressão.

$\log E(Y)=\log (T)+\beta_{0}+\beta_{1} X_{1}+\ldots+\beta_{p} X_{p}$

Em que:

$E(Y)$ : é o número esperado de óbitos;

$X_{1}, X_{2}, \ldots, X_{p}$ : são as variáveis explicativas consideradas no modelo;

$b_{0}$ : é o intercepto;

$b_{1}, b_{2}, \ldots, b_{p}$ : são os coeficientes da regressão que representam os efeitos das variáveis explicativas. Por exemplo, $b_{1}$ mede a variação para cada mudança unitária em $\mathrm{X}_{1}$, sendo mantida todas as outras variáveis $\left(\mathrm{X}_{2}, \ldots, \mathrm{X}_{\mathrm{p}}\right)$ constantes - por exemplo: se, no modelo, $\mathrm{b}_{1}$ é igual a 0,8 e $\mathrm{X}_{1}$ representa anos de estudo, o aumento de uma unidade na variável $\mathrm{X}_{1}$ indica que o valor do número esperado de óbitos $\mathrm{E}\left(\mathrm{Y} \mid \mathrm{X}_{1}\right)$ aumentará 0,8 unidades $\left(\mathrm{b}_{1}\right)$, sendo mantidas todas as outras variáveis constantes;

$e^{b}$ : razão entre as taxas de mortalidade das categorias de uma variável em relação à categoria de referência;
T: é o tempo total de exposição ao risco de morte, calculado, neste artigo, a partir da diferença entre a idade na data da entrevista em 2000 e idade à morte (no caso dos indivíduos que faleceram) ou idade na data da entrevista em 2006 (no caso dos indivíduos que sobreviveram);

$\log (T)$ : é uma variável explicativa com coeficiente fixo e igual a um.

Com base em modelos univariados, investigou-se, inicialmente, a relação entre cada variável explicativa, isoladamente, e a mortalidade dos idosos. As variáveis explicativas que apresentaram probabilidade de significância (valor de p) inferior a 0,20 foram consideradas elegíveis para compor os modelos de regressão de Poisson multivariados. Segundo alguns autores 6,17, esse procedimento é uma alternativa que deve ser considerada para se evitar que variáveis explicativas importantes sejam excluídas ou que variáveis de confusão sejam incluídas no modelo multivariado. No contexto deste artigo, esse procedimento foi adotado com o objetivo de tornar a escolha das variáveis explicativas, feita, inicialmente, a partir da literatura, mais parcimoniosa segundo um critério estatístico. A partir da aplicação desse critério, foram excluídas, da lista inicial, duas variáveis para os homens (se tem moradia própria e estado nutricional) e uma variável para as mulheres (hábito de fumar).

Escolhidas as variáveis candidatas para as análises multivariadas, três modelos foram ajustados para homens e mulheres separadamente. Tendo em vista o objetivo central deste trabalho, as variáveis estado marital e idade foram mantidas em todos os modelos multivariados, independente da sua significância estatística. No primeiro modelo, além do estado marital e da idade, foram acrescentadas as variáveis que representam condições de saúde dos idosos. Na sequência, no segundo modelo, foram adicionadas as variáveis econômicas e sociais. Por fim, o modelo 3 representa o modelo completo, e nele são consideradas tanto as variáveis socioeconômicas, como as de saúde e de estilo de vida. A cada passo, ao adicionar variáveis até se chegar ao modelo completo, pretende-se vislumbrar, com maior clareza, quais as características dos idosos que mais contribuem para explicar a associação entre estado marital e mortalidade. Nenhuma interação foi incluída nos modelos devido ao tamanho reduzido da amostra.

Para detectar a existência de multicolinearidade (dependência linear perfeita ou aproximada entre, pelo menos, duas variáveis explicativas), em cada um dos modelos multivariados ajustados, analisou-se a matriz de correlação entre os pares de coeficientes estimados. Nessas matrizes, correlações elevadas (geralmente supe- 
riores a 0,80-0,85) indicam um possível problema de colinearidade ${ }^{18}$. Para cada modelo estimado, as respectivas matrizes foram analisadas, e não foram detectados sinais importantes de colinearidade. Além disso, examinou-se a multicolinearidade por meio do VIF (Variance Inflation Factor). Geralmente, o VIF é indicativo de problemas de multicolinearidade se apresentar valores maiores que 10. Em todos os modelos estimados, essa medida não foi superior a 5 .

\section{Resultados}

\section{Análise descritiva}

Na Tabela 2, é apresentada a distribuição relativa dos idosos da amostra analisada, por sexo, segundo cada uma das variáveis independentes e sua condição de sobrevivência em 2006. Entre os homens que morreram durante o período de observação, verifica-se, relativamente aos que sobreviveram, uma maior proporção de indivíduos solteiros e viúvos (21\% contra $12 \%)$. Além disso, há, entre os que morreram, uma maior prevalência de idosos fumantes e que não praticavam atividade física, bem como idosos com saúde autorreportada regular ou ruim, com, pelo menos, uma limitação funcional, uma doença crônica e sem seguro de saúde. Observa-se, também, uma maior proporção de indivíduos de baixo status socioeconômico entre os mortos.

No que diz respeito às mulheres, verifica-se, assim como acontece com os homens, uma menor proporção de casadas entre as idosas que morreram, comparativamente às que sobreviveram ( $28 \%$ contra $46 \%$ ), embora o padrão de distribuição de divorciados/separados por condição de sobrevivência seja distinto entre homens e mulheres. Observa-se, também, entre as mulheres que morreram, uma maior prevalência de indivíduos mais velhos e com saúde autorreferida relativamente pior, qualquer que seja a variável examinada. Finalmente, como observado na amostra masculina, verifica-se uma relação negativa entre mortalidade e status socioeconômico.

\section{Resultados dos modelos multivariados}

Em relação aos homens casados (Tabela 3), os idosos solteiros apresentam taxas de mortalidade $72,6 \%$ maiores $(\mathrm{p}<0,05)$. Além disso, verifica-se que ser solteiro se mantém como um fator independentemente associado à mortalidade, mesmo após serem incluídas as variáveis que representam condições de saúde, aspectos econômicos e sociais e de estilo de vida. No ter- ceiro modelo, que considera todas as variáveis independentes, a taxa de mortalidade dos idosos solteiros é $61 \%$ maior que a taxa de mortalidade observada para os casados ( $p<0,05)$. Embora os coeficientes do modelo sugiram que as taxas de mortalidade também são mais altas para os homens viúvos, a diferença em relação aos homens casados não é estatisticamente significativa. Ao mesmo tempo, estima-se uma taxa de mortalidade entre os divorciados/separados que é quase a metade da taxa de mortalidade dos casados $(\mathrm{p}=0,046)$.

Entre as mulheres (Tabela 4), observam-se taxas de mortalidade relativamente maiores para as divorciadas/separadas (93\%) e viúvas (38\%) em comparação às casadas $(\mathrm{p}<0,05)$. A inclusão das variáveis de saúde, status social e econômico e de estilo de vida reduz a razão das taxas de mortalidade e sua significância estatística, mas, ainda assim, permanecem diferenças substantivas. No terceiro modelo, que considera todas as variáveis independentes, a razão de taxas de mortalidade torna-se igual a $1,81(\mathrm{p}<0,05)$ para as divorciadas/separadas e $1,35(\mathrm{p}<0,10)$ para as viúvas, em comparação às mulheres casadas.

Em relação à associação das demais variáveis independentes com a mortalidade, observa-se, conforme demonstrado na literatura e sugerido pela análise descritiva, uma associação positiva e significativa entre mortalidade e idade tanto para os homens quanto para as mulheres. Com base nas Tabelas 3 e 4, nota-se, também, que os idosos que reportaram sua saúde como regular ou ruim, que declararam ter maior número de doenças crônicas diagnosticadas por médico e que reportaram alguma dificuldade para realizar atividades básicas da vida diária apresentam taxas de mortalidade significativamente maiores, relativamente aos indivíduos mais saudáveis. Os resultados da análise multivariada demonstram, ainda, que a relação entre mortalidade e condições econômicas entre os idosos de São Paulo não é estatisticamente significativa, quando são considerados controles para as características demográficas, condições de saúde e estilos de vida. Por outro lado, a mortalidade é significativamente menor entre as mulheres que tiveram participação comunitária. Por fim, são observadas, no terceiro modelo, uma associação significativa e positiva entre consumo de tabaco e mortalidade masculina e uma relação significativa e negativa entre atividade física e mortalidade tanto entre homens quanto entre mulheres. 
Distribuição relativa das características da amostra, segundo status de sobrevivência e sexo. Município de São Paulo, Brasil, 2000.

\begin{tabular}{|c|c|c|c|c|c|c|}
\hline \multirow[t]{2}{*}{ Variáveis } & \multicolumn{3}{|c|}{ Homens (\%) } & \multicolumn{3}{|c|}{ Mulheres (\%) } \\
\hline & $\begin{array}{l}\text { Sobreviventes } \\
\qquad(n=496)\end{array}$ & $\begin{array}{l}\text { Óbitos } \\
(n=340)\end{array}$ & $\begin{array}{c}\text { Total } \\
(\mathrm{n}=836)\end{array}$ & $\begin{array}{l}\text { Sobreviventes } \\
\qquad(n=859)\end{array}$ & $\begin{array}{l}\text { Óbitos } \\
(n=309)\end{array}$ & $\begin{array}{c}\text { Total } \\
(n=1.168)\end{array}$ \\
\hline \multicolumn{7}{|l|}{ Estado marital } \\
\hline Casado & 82 & 76 & 79 & 46 & 28 & 41 \\
\hline Divorciado/Separado & 6 & 3 & 5 & 10 & 12 & 11 \\
\hline Viúvo & 9 & 15 & 11 & 38 & 57 & 43 \\
\hline Solteiro & 3 & 6 & 4 & 5 & 4 & 5 \\
\hline \multicolumn{7}{|l|}{ Grupos de idade (anos) } \\
\hline $60-69$ & 67 & 48 & 62 & 61 & 35 & 57 \\
\hline 70-79 & 28 & 33 & 29 & 31 & 32 & 31 \\
\hline 80 e mais & 5 & 19 & 9 & 7 & 33 & 12 \\
\hline \multicolumn{7}{|l|}{ Saúde autorreportada } \\
\hline Excelente/Muito boa/Boa & 52 & 38 & 48 & 47 & 36 & 45 \\
\hline Regular/Ruim & 48 & 62 & 52 & 53 & 64 & 55 \\
\hline \multicolumn{7}{|l|}{ Número de doenças crônicas } \\
\hline Nenhuma & 31 & 16 & 27 & 20 & 8 & 17 \\
\hline $1-2$ & 53 & 61 & 55 & 55 & 55 & 56 \\
\hline 3 ou mais & 16 & 23 & 18 & 25 & 37 & 27 \\
\hline \multicolumn{7}{|l|}{ Presença de incapacidade (ABVD) } \\
\hline Nenhuma & 90 & 74 & 85 & 81 & 59 & 78 \\
\hline Pelo menos 1 & 10 & 26 & 15 & 19 & 41 & 22 \\
\hline \multicolumn{7}{|l|}{ Nível de escolaridade (anos) } \\
\hline Nenhum & 15 & 24 & 18 & 20 & 36 & 23 \\
\hline $1-7$ & 61 & 61 & 60 & 64 & 56 & 62 \\
\hline 8 ou mais & 24 & 15 & 22 & 15 & 8 & 15 \\
\hline \multicolumn{7}{|c|}{ Tem seguro/plano privado de saúde? } \\
\hline $\operatorname{Sim}$ & 39 & 27 & 35 & 40 & 31 & 38 \\
\hline Não & 61 & 73 & 65 & 60 & 69 & 62 \\
\hline \multicolumn{7}{|l|}{ Tem casa própria? } \\
\hline Sim & 84 & 79 & 81 & 82 & 72 & 77 \\
\hline Não & 16 & 21 & 19 & 18 & 28 & 23 \\
\hline \multicolumn{7}{|l|}{ Uso de serviços de saúde } \\
\hline Usou & 77 & 83 & 79 & 86 & 88 & 86 \\
\hline Não usou & 23 & 17 & 21 & 14 & 12 & 14 \\
\hline \multicolumn{7}{|l|}{ Estado nutricional } \\
\hline Bem nutrido & 95 & 96 & 96 & 93 & 85 & 91 \\
\hline Não está bem nutrido & 5 & 4 & 4 & 7 & 7 & 9 \\
\hline \multicolumn{7}{|c|}{ Teve participação comunitária no último ano? } \\
\hline Não participou & 71 & 84 & 75 & 68 & 86 & 72 \\
\hline Participou & 29 & 16 & 25 & 32 & 14 & 28 \\
\hline \multicolumn{7}{|l|}{ Hábito de fumar } \\
\hline Fuma atualmente & 19 & 34 & 23 & 11 & 13 & 11 \\
\hline Já fumou, mas não fuma mais & 52 & 45 & 51 & 17 & 21 & 19 \\
\hline Nunca fumou & 29 & 21 & 26 & 73 & 66 & 70 \\
\hline \multicolumn{7}{|l|}{ Uso de álcool } \\
\hline Não faz uso & 49 & 61 & 53 & 77 & 87 & 79 \\
\hline Faz uso & 51 & 39 & 47 & 23 & 13 & 21 \\
\hline
\end{tabular}

(continua) 
Tabela 2 (continuação)

\begin{tabular}{|c|c|c|c|c|c|c|}
\hline \multirow[t]{2}{*}{ Variáveis } & \multicolumn{3}{|c|}{ Homens (\%) } & \multicolumn{3}{|c|}{ Mulheres (\%) } \\
\hline & $\begin{array}{l}\text { Sobreviventes } \\
\qquad(n=496)\end{array}$ & $\begin{array}{l}\text { Óbitos } \\
(n=340)\end{array}$ & $\begin{array}{c}\text { Total } \\
(\mathrm{n}=836)\end{array}$ & $\begin{array}{l}\text { Sobreviventes } \\
\qquad(\mathrm{n}=859)\end{array}$ & $\begin{array}{l}\text { Óbitos } \\
(\mathrm{n}=309)\end{array}$ & $\begin{array}{c}\text { Total } \\
(n=1.168)\end{array}$ \\
\hline \multicolumn{7}{|c|}{ Prática de atividade física } \\
\hline $\operatorname{Sim}$ & 35 & 19 & 30 & 29 & 10 & 24 \\
\hline Não & 65 & 81 & 70 & 71 & 90 & 76 \\
\hline
\end{tabular}

ABVD: atividades básicas da vida diária.

Fonte: Estudo SABE, 2000 e 2006.

Nota: A participação relativa de cada categoria nas variáveis analisadas foi obtida após a expansão da amostra. Para os idosos do sexo masculino: 49 (5,8\%) deixaram em branco as informações sobre doenças crônicas diagnosticadas por médico; 8 (0,9\%) não declararam nível de escolaridade; 1 (0,1\%) não informou se a casa que habitava era própria; e $10(1,2 \%)$ não relataram o seu estado nutricional. Já entre as idosas: 1 (0,08\%) apresentou estado marital em branco; 5 $(0,4 \%)$ não autoavaliaram a sua saúde; 78 (6,6\%) deixaram em branco as informações sobre doenças crônicas diagnosticadas por médico; 1 (0,08\%) apresentou informações sobre incapacidade em branco; $9(0,7 \%)$ não declararam nível de escolaridade; $1(0,08 \%)$ não informou se tem seguro privado de saúde ou se fez uso de serviços de saúde ou se teve participação comunitária ou se fez uso de álcool; 2 (0,1\%) não informaram se a casa é própria; 25 ( $2,1 \%)$ não relataram o seu estado nutricional; para 17 (1,4\%), não foi possível obter o índice de frequência mediana de atenção; e 2 (0,1\%) não relataram se fumavam ou se praticavam exercício regularmente.

Tabela 3

Estimativas para os parâmetros dos modelos de Poisson multivariados. Mortalidade masculina. Município de São Paulo, Brasil, $2000-2006$.

\begin{tabular}{|c|c|c|c|c|c|c|c|c|c|}
\hline \multirow[t]{2}{*}{ Variáveis } & \multicolumn{3}{|c|}{ Modelo 1} & \multicolumn{3}{|c|}{ Modelo 2} & \multicolumn{3}{|c|}{ Modelo 3} \\
\hline & Coeficiente & RTM & Valor de p & Coeficiente & RTM & Valor de $p$ & Coeficiente & RTM & Valor de $p$ \\
\hline \multicolumn{10}{|l|}{ Características demográficas } \\
\hline \multicolumn{10}{|l|}{ Estado marital } \\
\hline Casado & - & 1,000 & & - & 1,000 & & - & 1,000 & \\
\hline Divorciado/Separado & $-0,486$ & 0,615 & 0,191 & $-0,555$ & 0,574 & 0,122 & $-0,646$ & 0,524 & 0,046 * \\
\hline Viúvo & 0,172 & 1,187 & 0,386 & 0,170 & 1,185 & 0,389 & 0,151 & 1,163 & 0,444 \\
\hline Solteiro & 0,546 & 1,726 & 0,036 * & 0,463 & 1,589 & $0,082 * \star$ & 0,478 & 1,613 & 0,045 * \\
\hline \multicolumn{10}{|l|}{ Grupos de idade (anos) } \\
\hline $60-69$ & - & 1,000 & & - & 1,000 & & - & 1,000 & \\
\hline $70-79$ & 0,538 & 1,713 & 0,011 & 0,523 & 1,687 & $0,012 * \star \star$ & 0,573 & 1,774 & $0,004 * \star \star$ \\
\hline 80 e mais & 1,274 & 3,576 & $<0,001 \star \star \star$ & 1,225 & 3,405 & $<0,001 \star \star \star$ & 1,299 & 3,667 & 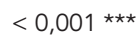 \\
\hline \multicolumn{10}{|l|}{ Condições de saúde } \\
\hline \multicolumn{10}{|l|}{ Saúde autorreportada } \\
\hline Excelente/Muito boa/Boa & - & 1,000 & & - & 1,000 & & - & 1,000 & \\
\hline Regular/Ruim & 0,291 & 1,337 & 0,046 * & 0,220 & 1,246 & 0,133 & 0,207 & 1,231 & 0,152 \\
\hline \multicolumn{10}{|l|}{ Número de doenças crônicas } \\
\hline Nenhuma & - & 1,000 & & - & 1,000 & & - & 1,000 & \\
\hline $1-2$ & 0,579 & 1,785 & $0,004 * \star \star$ & 0,596 & 1,816 & $0,002 * \star \star$ & 0,596 & 1,815 & $0,002 * \star \star *$ \\
\hline 3 ou mais & 0,575 & 1,777 & 0,016 * & 0,586 & 1,797 & $0,013 * \star \star$ & 0,653 & 1,921 & $0,006 * \star \star$ \\
\hline \multicolumn{10}{|c|}{ Presença de incapacidade (ABVD) } \\
\hline Nenhuma & - & 1,000 & & - & 1,000 & & - & 1,000 & \\
\hline Pelo menos uma & 0,477 & 1,611 & $0,003 * \star \star$ & 0,398 & 1,489 & $0,007 * \star \star$ & 0,313 & 1,367 & 0,037 * \\
\hline \multicolumn{10}{|l|}{ Status socioeconômico } \\
\hline \multicolumn{10}{|l|}{ Nível de escolaridade (anos) } \\
\hline Nenhum & & & & - & 1,000 & & - & 1,000 & \\
\hline $1-7$ & & & & $-0,066$ & 0,936 & 0,667 & 0,017 & 1,017 & 0,909 \\
\hline 8 ou mais & & & & $-0,227$ & 0,797 & 0,457 & $-0,115$ & 0,891 & 0,701 \\
\hline
\end{tabular}

(continua) 
Tabela 3 (continuação)

\begin{tabular}{|c|c|c|c|c|c|c|c|c|c|}
\hline \multirow[t]{2}{*}{ Variáveis } & \multicolumn{3}{|c|}{ Modelo 1} & \multicolumn{3}{|c|}{ Modelo 2} & \multicolumn{3}{|c|}{ Modelo 3} \\
\hline & Coeficiente & RTM & Valor de $p$ & Coeficiente & RTM & Valor de $\mathrm{p}$ & Coeficiente & RTM & Valor de $\mathrm{p}$ \\
\hline \multicolumn{10}{|l|}{ Tem seguro/plano privado de saúde? } \\
\hline $\operatorname{Sim}$ & & & & - & 1,000 & & - & 1,000 & \\
\hline Não & & & & 0,320 & 1,378 & 0,161 & 0,202 & 1,223 & 0,395 \\
\hline \multicolumn{10}{|l|}{ Uso de serviços de saúde } \\
\hline Usou & & & & - & 1,000 & & - & 1,000 & \\
\hline Não usou & & & & $-0,015$ & 0,985 & 0,932 & 0,022 & 1,022 & 0,900 \\
\hline \multicolumn{10}{|l|}{ Meio social } \\
\hline \multicolumn{10}{|c|}{ Teve participação comunitária no último ano? } \\
\hline Não participou & & & & - & 1,000 & & - & 1,000 & \\
\hline Participou & & & & $-0,444$ & 0,642 & 0,019 * & $-0,289$ & 0,749 & 0,150 \\
\hline \multicolumn{10}{|l|}{ Estilo de vida } \\
\hline \multicolumn{10}{|l|}{ Hábito de fumar } \\
\hline Fuma atualmente & & & & & & & - & 1,000 & \\
\hline Já fumou, mas não fuma mais & & & & & & & $-0,674$ & 0,510 & $<0,001 * * *$ \\
\hline Nunca fumou & & & & & & & $-0,721$ & 0,486 & $0,002 * \star \star$ \\
\hline \multicolumn{10}{|l|}{ Uso de álcool } \\
\hline Não faz uso & & & & & & & - & 1,000 & \\
\hline Faz uso & & & & & & & $-0,143$ & 0,867 & 0,334 \\
\hline \multicolumn{10}{|l|}{ Prática de atividade física } \\
\hline $\operatorname{Sim}$ & & & & & & & - & 1,000 & \\
\hline Não & & & & & & & 0,407 & 1,502 & 0,021 * \\
\hline Tamanho da amostra & & 787 & & & 779 & & & 779 & \\
\hline Constante & $-4,19$ & & $<0,001$ *** & $-4,17$ & & $<0,001$ *** & $-3,95$ & & $<0,001$ *** \\
\hline Valor de F & & 16,77 & & & 10,66 & & & 13,69 & \\
\hline Valor de $p$ & & $<0,001$ & & & $<0,001$ & & & $<0,001$ & \\
\hline
\end{tabular}

ABVD: atividades básicas da vida diária; RTM: razão entre as taxas de mortalidade.

Fonte: Estudo SABE, 2000 e 2006.

* $p<0,05$;

** $p<0,10$;

*** $p<0,01$.

Tabela 4

Estimativas para os parâmetros dos modelos de Poisson multivariados. Mortalidade feminina. Município de São Paulo, Brasil, 2000-2006.

\begin{tabular}{|c|c|c|c|c|c|c|c|c|c|}
\hline \multirow[t]{2}{*}{ Variáveis } & \multicolumn{3}{|c|}{ Modelo 1} & \multicolumn{3}{|c|}{ Modelo 2} & \multicolumn{3}{|c|}{ Modelo 3} \\
\hline & Coeficiente & RTM & Valor de $p$ & Coeficiente & RTM & Valor de $p$ & Coeficiente & RTM & Valor de $p$ \\
\hline \multicolumn{10}{|c|}{ Características demográficas } \\
\hline \multicolumn{10}{|c|}{ Estado marital } \\
\hline Casado & - & 1,000 & & - & 1,000 & & - & 1,000 & \\
\hline Divorciado/Separado & 0,659 & 1,933 & 0,018 * & 0,540 & 1,716 & $0,069 * \star$ & 0,598 & 1,819 & 0,042 * \\
\hline Viúvo & 0,392 & 1,480 & 0,024 * & 0,333 & 1,395 & 0,055 * & 0,303 & 1,354 & 0,085 ** \\
\hline Solteiro & 0,010 & 1,010 & 0,968 & 0,046 & 1,047 & 0,864 & 0,048 & 1,049 & 0,857 \\
\hline \multicolumn{10}{|l|}{ Grupos de idade (anos) } \\
\hline $60-69$ & - & 1,000 & & - & 1,000 & & - & 1,000 & \\
\hline $70-79$ & 0,517 & 1,676 & 0,020 * & 0,430 & 1,537 & 0,053 * & 0,421 & 1,524 & 0,059 ** \\
\hline 80 e mais & 1,469 & 4,344 & $<0,001 * \star \star$ & 1,409 & 4,090 & $<0,001 * * *$ & 1,321 & 3,745 & $<0,001$ *** \\
\hline
\end{tabular}

(continua) 
Tabela 4 (continuação)

\begin{tabular}{|c|c|c|c|c|c|c|c|c|c|}
\hline \multirow[t]{2}{*}{ Variáveis } & \multicolumn{3}{|c|}{ Modelo 1} & \multicolumn{3}{|c|}{ Modelo 2} & \multicolumn{3}{|c|}{ Modelo 3} \\
\hline & Coeficiente & RTM & Valor de $p$ & Coeficiente & RTM & Valor de $p$ & Coeficiente & RTM & Valor de $p$ \\
\hline \multicolumn{10}{|l|}{ Condições de saúde } \\
\hline \multicolumn{10}{|l|}{ Saúde autorreportada } \\
\hline Excelente/Muito boa/Boa & - & 1,000 & & - & 1,000 & & - & 1,000 & \\
\hline Regular/Ruim & 0,037 & 1,038 & 0,831 & $-0,034$ & 0,967 & 0,855 & $-0,111$ & 0,895 & 0,565 \\
\hline \multicolumn{10}{|l|}{ Número de doenças crônicas } \\
\hline Nenhuma & - & 1,000 & & - & 1,000 & & - & 1,000 & \\
\hline $1-2$ & 0,741 & 2,098 & $0,002 * \star \star *$ & 0,774 & 2,169 & 0,002 *** & 0,777 & 2,176 & 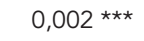 \\
\hline 3 ou mais & 0,912 & 2,488 & $0,001 * \star \star$ & 0,981 & 2,666 & $0,001 * \star \star$ & 0,928 & 2,530 & $0,001 * \star \star$ \\
\hline \multicolumn{10}{|c|}{ Presença de incapacidade (ABVD) } \\
\hline Nenhuma & - & 1,000 & & - & 1,000 & & - & 1,000 & \\
\hline Pelo menos uma & 0,594 & 1,812 & $<0,001 \star \star \star$ & 0,508 & 1,662 & $0,001 * \star \star$ & 0,438 & 1,550 & $0,002 * \star \star$ \\
\hline \multicolumn{10}{|l|}{ Status socioeconômico } \\
\hline \multicolumn{10}{|l|}{ Nível de escolaridade (anos) } \\
\hline Nenhum & & & & - & 1,000 & & - & 1,000 & \\
\hline $1-7$ & & & & $-0,249$ & 0,779 & $0,107 * \star$ & $-0,205$ & 0,814 & 0,194 \\
\hline 8 ou mais & & & & $-0,275$ & 0,760 & 0,365 & $-0,150$ & 0,861 & 0,621 \\
\hline \multicolumn{10}{|c|}{ Tem seguro/plano privado de saúde? } \\
\hline $\operatorname{Sim}$ & & & & - & 1,000 & & - & 1,000 & \\
\hline Não & & & & 0,072 & 1,075 & 0,608 & 0,066 & 1,068 & 0,631 \\
\hline \multicolumn{10}{|l|}{ Tem casa própria? } \\
\hline Sim & & & & - & 1,000 & & - & 1,000 & \\
\hline Não & & & & 0,246 & 1,279 & 0,132 & 0,234 & 1,264 & 0,163 \\
\hline \multicolumn{10}{|l|}{ Estado nutricional } \\
\hline Bem nutrido & & & & - & 1,000 & & - & 1,000 & \\
\hline Não está bem nutrido & & & & 0,312 & 1,366 & 0,145 & 0,279 & 1,322 & 0,180 \\
\hline \multicolumn{10}{|l|}{ Meio social } \\
\hline \multicolumn{10}{|c|}{ Teve participação comunitária no último ano? } \\
\hline Não participou & & & & - & 1,000 & & - & 1,000 & \\
\hline Participou & & & & $-0,600$ & 0,549 & $0,004 * \star \star$ & $-0,589$ & 0,555 & $0,005 * \star \star$ \\
\hline \multicolumn{10}{|l|}{ Estilo de vida } \\
\hline \multicolumn{10}{|l|}{ Uso de álcool } \\
\hline Não faz uso & & & & & & & - & 1,000 & \\
\hline Faz uso & & & & & & & $-0,388$ & 0,678 & $0,075 \star \star$ \\
\hline \multicolumn{10}{|l|}{ Prática de atividade física } \\
\hline Sim & & & & & & & - & 1,000 & \\
\hline Não & & & & & & & 0,731 & 2,078 & $0,003 * \star \star$ \\
\hline Tamanho da amostra & & 1.083 & & & 1.044 & & & 1.041 & \\
\hline Constante & $-5,26$ & & $<0,001 * \star \star$ & $-4,95$ & & $<0,001 \star \star \star *$ & $-5,42$ & & $<0,001 \star \star \star *$ \\
\hline Valor de F & & 18,34 & & & 13,70 & & & 13,45 & \\
\hline Valor de $p$ & & $<0,001$ & & & $<0,001$ & & & $<0,001$ & \\
\hline
\end{tabular}

ABVD: atividades básicas da vida diária; RTM: razão entre as taxas de mortalidade.

Fonte: Estudo SABE, 2000 e 2006.

${ }^{*} p<0,05$;

${ }^{* *} p<0,10$;

${ }^{\star \star \star} p<0,01$. 


\section{Discussão}

Diversos estudos na literatura sugerem que a mortalidade entre os idosos não casados é maior do que entre os idosos casados. No entanto, quando se examinam os diferenciais de mortalidade entre os grupos de idosos não casados, os resultados variam segundo a população analisada 3,4,5,6,7,8,9. Os resultados apresentados neste artigo, embora confirmem o maior risco de morte dos idosos não casados, apontam para uma diferença importante nos diferenciais de mortalidade por estado marital entre homens e mulheres. No caso dos idosos paulistanos do sexo masculino, os solteiros formam o grupo mais vulnerável, ao passo que, entras as idosas, são as divorciadas e separadas que apresentam as taxas mais altas de mortalidade. Em relação à população masculina, resultado semelhante foi observado por $\mathrm{Hu} \&$ Goldman ${ }^{3}$, para Portugal e Japão, em estudo que teve como objetivo analisar a mortalidade relativa por estado marital em 16 países desenvolvidos, no período de 1950 a 1980 . As estimativas também são consistentes com as que Valkonen et al. 19 encontraram para o Canadá e para a maioria dos países da Europa Ocidental, nas décadas de 1980 e 1990, bem como com as estimativas de Gomes et al. 20 para Porto Rico, no período de 2002-2007.

Segundo Lillard \& Panis 4 e Goldman \& Hu 7, o excesso de mortalidade entre os idosos solteiros pode ser explicado tanto pela seletividade do casamento quanto por mecanismos de proteção. Ou seja, o maior nível de mortalidade entre os idosos solteiros seria fruto tanto da redução na chance de casamento para indivíduos de menor status socioeconômico e em piores condições de saúde quanto pela proteção que o casamento oferece em termos de redução da vulnerabilidade socioeconômica e melhoria nos hábitos de vida e de saúde. Infelizmente, o simples controle desses fatores nos modelos analisados não permite definir qual é a verdadeira direção da causalidade no caso de São Paulo.

Ainda em relação aos homens, as menores taxas de mortalidade observadas entre divorciados e separados, comparativamente aos idosos casados, poderiam ser explicadas por uma combinação de efeitos de seleção - indivíduos mais saudáveis teriam maior propensão à separação - com efeitos de proteção, representados por benefícios adquiridos e acumulados ao longo do período vivido em matrimônio.

Ao contrário dos homens, o fim do matrimônio, por meio de separação, divórcio ou viuvez, é o fator responsável por reduzir a sobrevivência de mulheres idosas em São Paulo. Resultado semelhante foi encontrado por Hemstrom 8 em análise para a Suécia e por Martikainen \& Valkonen 21 em estudo realizado para a Finlândia. Hemstrom ${ }^{8}$ destaca que não é apenas a transição para outro estado marital que faz da dissolução do casamento um evento estressante, com efeitos negativos nocivos sobre a longevidade, mas, também, a mudança para um novo estilo de vida que exige adaptação às novas condições como redução de suporte social e perda material. Nesse sentido, é possível que, no caso de São Paulo, os efeitos distintos da dissolução do casamento sobre a mortalidade de homens e mulheres reflitam a menor autonomia financeira das mulheres em relação aos homens.

Os resultados apresentados neste trabalho contribuem para um melhor entendimento sobre o padrão de mortalidade da população idosa brasileira, em particular, seus diferencias entre subgrupos demográficos. Como apontam Campos 17 e Santos et al. ${ }^{22}$, em países em desenvolvimento, onde há escassez de informações, pesquisas como o Estudo SABE são centrais para a investigação dos fatores associados à mortalidade, o que, em última instância, favorece a formulação de políticas públicas direcionadas aos idosos mais vulneráveis. No entanto, a análise apresentada neste artigo não está livre de limitações. A primeira delas está relacionada com a natureza da amostra da pesquisa. O Estudo SABE não entrevista os idosos institucionalizados, o que implica em uma amostra seletiva de indivíduos mais saudáveis do que a média da população na mesma faixa etária 23 . De qualquer forma, como salientam Lima-Costa \& Barreto 24 , esse tipo de viés é mais acentuado entre os idosos mais velhos, já que a probabilidade de institucionalização aumenta com a idade e em comunidades com maior grau de institucionalização. Considerando que os idosos institucionalizados no Município de São Paulo constituem uma fração reduzida da população com 60 anos e mais, espera-se, portanto, que sua ausência afete pouco os resultados deste trabalho.

Outra limitação deste estudo é a possível existência de viés de seletividade na amostra em função da exclusão de indivíduos ao longo do período de observação ou pelo fato de a pesquisa não considerar os indivíduos que morreram antes dos 60 anos de idade. No primeiro caso, como já foi discutido na seção metodológica, a perda de entrevistados, no período entre 2000 e 2006 , equivale a pouco mais de $6 \%$ da amostra original, e sua exclusão não alterou a distribuição das variáveis utilizadas nos modelos. Em relação à segunda fonte de viés, conforme Monteverde et al. 25 argumentam, espera-se que esse efeito seja pequeno, já que uma parte considerável das coortes atuais de idosos no Brasil se beneficiou 
das reduções ocorridas, desde a primeira metade do século passado, na mortalidade infantil e infanto-juvenil.

Com relação às variáveis utilizadas nos modelos, é importante fazer a ressalta que as informações do SABE são coletadas por meio de autorrelato, e, portanto, as respostas podem ser influenciadas por fatores culturais, de memória, pela escolaridade e por questões emocionais e psicológicas. Possíveis problemas de precisão na qualidade dessas informações podem atenuar ou fortalecer os efeitos dos parâmetros estimados nos modelos. Além disso, todas as análises foram realizadas considerando as informações coletadas em 2000, na linha de base da pesquisa. É possível que, durante o período de observação, alguns dos participantes da pesquisa tenham mudado de estado marital, alterado seus hábitos de vida, ou experimentado novos eventos de saúde. Não é possível determinar qual seria o impacto dessas mudanças sobre os resultados deste trabalho, embora, em análise cujas estimativas não foram apresentadas no artigo, não observamos mudanças significativas na situação conjugal em 2006 em relação a 2000 para os sobreviventes. Ressalta-se, no entanto, que o período de observação não é muito longo, e o mais provável é que algumas dessas variáveis não tenham sofrido alterações relevantes entre 2000 e 2006. Portanto, conforme sugerido em outros trabalhos da literatura internacional que utilizaram metodologia semelhante 9,10, dificilmente os resultados apresentados seriam alterados significativamente.

Outro ponto que merece ser destacado é o fato de a categoria "casado" no SABE incluir tanto as uniões formais quanto as consensuais. Embora não seja possível separar essas duas categorias, deve-se ressaltar que, entre os idosos, a prevalência de uniões consensuais é mais baixa do que no restante da população, não atingindo $2 \%$ em 2000 26. Além disso, a hipótese de que essas duas formas de casamento têm efeitos distintos sobre a sobrevivência não parece plausível, dado o grau de estabilidade que é atribuído à união consensual na sociedade brasileira.

Por fim, vale ressaltar que os resultados apresentados neste artigo se restringem aos idosos residentes no Município de São Paulo, no período de 2000-2006. Tendo em vista a heterogeneidade da população brasileira, possíveis generalizações das estimativas aqui apresentadas devem considerar a provável existência de variações nos mecanismos de proteção e seleção em cada região do país. Uma vez que o Estudo SABE também foi realizado em outros seis países da América Latina 17, a elaboração de análises comparativas sobre o tema permitiria melhorar a compreensão dos determinantes da mortalidade dos idosos, especialmente em países em desenvolvimento.

\section{Resumo}

Este trabajo tiene como objetivo analizar la asociación entre el estado civil y la mortalidad, entre la población de 60 años o más que vive en São Paulo, Brasil. Para alcanzar ese objetivo, se utilizaron los datos del Estudio $S A B E$ (Salud, Bienestar y Envejecimiento), realizado en 2000 y 2006, y los modelos de regresión de Poisson se estimaron teniendo en cuenta la variación tiempo en el riesgo de muerte. En general, los resultados indican que, entre los ancianos de sexo masculino en São Paulo, la tasa de mortalidad de solteros es un $61 \%$ más alta que la tasa de mortalidad observada para los ca- sados. A su vez, la separación/divorcio o viudez parece aumentar el riesgo de muerte de las mujeres ancianas. En general, las ancianas separadas y viudas presentaron tasas de mortalidad un $82 \%$ y un $35 \%$ mayores que la observada para las casadas. Se espera que este trabajo contribuya a una mejor comprensión de los factores asociados con la supervivencia de los ancianos, además de apoyar las políticas de salud dirigidas a este contingente de población.

Mortalidad; Anciano; Estado Civil 


\section{Colaboradores}

M. M. F. Gomes participou da concepção do projeto, análise e interpretação dos dados e redação do artigo. Também revisou criticamente o conteúdo intelectual do artigo e aprovou a versão final a ser publicada. C. M. Turra orientou o trabalho, participou da concepção do projeto e da redação do artigo. Também revisou criticamente o conteúdo intelectual do artigo e aprovou a versão final a ser publicada. M. G. B. Fígoli orientou o trabalho, participou da concepção do projeto e da redação do artigo. Também revisou criticamente o conteúdo intelectual do artigo e aprovou a versão final a ser publicada. Y. A. O. Duarte participou da redação do artigo. Também revisou criticamente o conteúdo intelectual do artigo e aprovou a versão final a ser publicada. M. L. Lebrão participou da redação do artigo. Também revisou criticamente o conteúdo intelectual do artigo e aprovou a versão final a ser publicada.

\section{Referências}

1. Vaupel JW. Demographic analysis of aging and longevity. Am Econ Rev 1998; 88:242-7.

2. Camarano AA, Kanso S, Mello JL. Quão além dos 60 poderão viver os idosos brasileiros? In: Camarano AM, organizador. Os novos idosos brasileiros: muito além dos 60 ? Rio de Janeiro: Instituto de Pesquisa Econômica Aplicada; 2004. p. 411-26.

3. Hu Y, Goldman N. Mortality differentials by marital status: an international comparison. Demography 1990; 27:233-50.

4. Lillard LA, Panis CW. Marital status and mortality: the role of health. Demography 1996; 33:313-27.

5. Lusyne P, Page H, Lievens J. Mortality following conjugal bereavement, Belgium 1991-96: the unexpected effect of education. Popul Stud 2001; 55:281-9.

6. Murphy M, Grundy E, Kalogirou S. The increase in marital status differences in mortality up to the oldest age in seven European countries, 1990-99. Popul Stud 2007; 61:287-98.

7. Goldman N, Hu Y. Excess mortality among the unmarried: a case study of Japan. Soc Sci Med 1993; 36:533-46.

8. Hemstrom O. Is marriage dissolution linked to differences in mortality risks for men and women? J Marriage Fam 1996; 58:366-78.

9. Johnson NJ, Backlund E, Sorlie PD, Loveless CA. Marital status and mortality: the national longitudinal mortality study. Ann Epidemiol 2000; 10: 224-38.

10. Goldman N, Korenman S, Weinstein R. Marital status and health among the elderly. Soc Sci Med 1995; 40:1717-30.

11. Van Den Berg GJ, Lindeboom M, Portrait F. Conjugal bereavement effects on health and mortality at advanced ages. Bonn: IZA Institute; 2006. (IZA Discussion Paper, 2358).

12. Medeiros M, Osório RG. Mudanças na composição dos arranjos domiciliares no Brasil - 1978 a 1998. Rev Bras Estud Popul 2000; 17:67-85.

13. Manzoli L, Villari P, M Pirone G, Boccia A. Marital status and mortality in the elderly: a systematic review and meta-analysis. Soc Sci Med 2007; 64: 77-94.

14. Silva NN. Aspectos metodológicos: processo de amostragem. In: Lebrão ML, Duarte YAO, organizadoras. O projeto SABE no Município de São Paulo: uma abordagem inicial. Brasília: Organização Pan-Americana da Saúde; 2003. p. 47-57.

15. Lebrão ML, Duarte YAO, Santos JLF, Laurenti R. Evolução nas condições de vida e saúde da população idosa do Município de São Paulo. São Paulo Perspect 2008; 22:30-45. 
16. Jewell NP, Hubbard A. Analysis of longitudinal studies in epidemiology. Berkeley: University of California Press; 2006.

17. Campos NOB. Os determinantes das condições de saúde dos idosos do Município de São Paulo em uma perspectiva de ciclo de vida [Tese de Doutorado]. Belo Horizonte: Centro de Desenvolvimento e Planejamento Regional, Universidade Federal de Minas Gerais; 2009.

18. Hamilton LC. Statistics with STATA: updated for version 9. Belmont: Thomson Books; 2006.

19. Valkonen T, Martikainen P, Blomgren J. Increasing excess mortality among nonmarried elderly people in developed countries. Demogr Res 2004; 2:306-30.

20. Gomes MMF, Gonzaga MR, Queiroz BL. Mortalidade segundo estado marital entre os idosos portoriquenhos. In: Anais do IV Congresso da Associação Latino-americana de População [CD-ROM]. La Habana: Associação Latino-americana de População; 2010.

21. Martikainen P, Valkonen T. Mortality after the death of a spouse rates and causes of death in a large Finnish cohort. Am J Public Health 1996; 86:1087-93.

22. Santos JL, Oliveira ML, de Souza MR, et al. O meio rural e a origem do idoso: a saúde e a morte na cidade (resultados do Estudo SABE 2000-2006). In: Anais do XVII Encontro Nacional de Estudos Populacionais [CD-ROM]. Caxambu: Associação Brasileira de Estudos Populacionais; 2010.
23. Cerqueira MBR. Envelhecimento populacional e população institucionalizada: um estudo de caso dos asilos do Município de Montes Claros [Dissertação de Mestrado]. Belo Horizonte: Centro de Desenvolvimento e Planejamento Regional, Universidade Federal de Minas Gerais; 2003.

24. Lima-Costa MF, Barreto SM. Tipos de estudos epidemiológicos: conceitos básicos e aplicações na área do envelhecimento. Epidemiol Serv Saúde 2003; 12:189-201.

25. Monteverde M, Noronha K, Palloni A. Effect of early conditions on disability among elderly in Latin America and the Caribbean. Madison: University of Wisconsin-Madison; 2011. (CDE Working Paper, $11)$.

26. Costa CS. Uniões informais no Brasil em 2000: uma análise sob a ótica da mulher [Dissertação de Mestrado]. Belo Horizonte: Centro de Desenvolvimento e Planejamento Regional, Universidade Federal de Minas Gerais; 2004.

Recebido em 04/Jun/2012

Versão final reapresentada em 16/Set/2012

Aprovado em 03/Out/2012 